\title{
Necrotising enterocolitis after neonatal cardiac catheterisation
}

\author{
D F DICKINSON, R W GALLOWAY, J L WILKINSON, AND R ARNOLD \\ Regional Paediatric Cardiothoracic Unit, Royal Liverpool Children's Hospital
}

SUMMARY Evidence of necrotising enterocolitis was found in 14 of 111 neonatal cardiac catheterisations. A close temporal relationship between investigation and the onset of necrotising enterocolitis was apparent in many cases but no predisposing factors could be identified.

Necrotising enterocolitis (NEC) is known to be an important cause of morbidity and mortality in neonatal intensive care units. ${ }^{2}$ It is seen mainly in preterm infants but has been reported in term ones including some with congenital heart disease. ${ }^{13}$ Recently Cooke et al. ${ }^{4}$ reported 3 cases which followed cardiac catheterisation and a causal or contributory relationship was suggested. Two clusters of cases of NEC occurring in this unit within a short period of time prompted us to review a representative group of infants who underwent cardiac catheterisation for evidence of this complication and to look for possible predisposing factors.

\section{Patients and methods}

One hundred and eleven consecutive infants who underwent cardiac catheterisation within the first month of life during the period January 1980 to October 1981 and survived for more than 24 hours were reviewed for evidence of NEC. The presence of frankly blood-stained stools and abdominal distension was accepted as clinical evidence of NEC, and confirmatory evidence from serial abdominal radiographs and peripheral blood count was sought in each patient. Infants who developed NEC were compared with those who did not, with respect to a number of factors using the exact probability test and Wilcoxon's rank sum test for statistical analysis.

\section{Results}

Fourteen $(13 \%)$ patients had evidence of NEC. The clinical and laboratory data from these patients are summarised in Table 1. Seven cases occurred in two clusters, the remaining 7 cases being distributed sporadically throughout the 22 -month period. Four patients died, 3 primarily as a result of NEC and one (Case 1, Table 1) mainly as a result of the cardiac problem. In this patient the first symptom of NEC preceded cardiac catheterisation by several hours; in the remaining patients the onset followed investigation by 1 to 13 days. Five patients underwent emergency surgery for their cardiac problem and all survived. In two (Cases 6 and 8, Table 1) surgery preceded the onset of NEC by 6 and 5 days, but in Case 13 operation was performed 3 days after the first symptom of NEC. Cases 11 and 12 underwent operation within 24 hours of cardiac catheterisation and NEC was diagnosed in the immediate postoperative period.

The illness varied in severity from a fairly minor disturbance with recovery within $\mathbf{4 8}$ hours to a major fulminating illness leading to death after 9 days of intensive treatment. All infants were treated by suspending oral feeding and the intravenous administration of gentamicin, penicillin, metronidazole, and appropriate electrolyte solution. The 2 infants who developed intestinal perforation did so as a terminal event and did not undergo laparotomy. At necropsy these infants had extensive NEC from the upper third of the ileum to the descending colon. No infant with NEC had evidence of either generalised systemic or localised gastrointestinal infection.

A comparison of possible predisposing factors in those who developed NEC and those who did not showed no statistically significant differences between the two groups (Table 2).

\section{Discussion}

Since this investigation was prompted by two clusters of cases it is probable that the prevalence of NEC reported is higher than the true prevalence of this complication among neonates with congenital heart disease. Nevertheless, it is clear that 
Table 1 Clinical details and evidence of necrotising enterocolitis in 14 patients

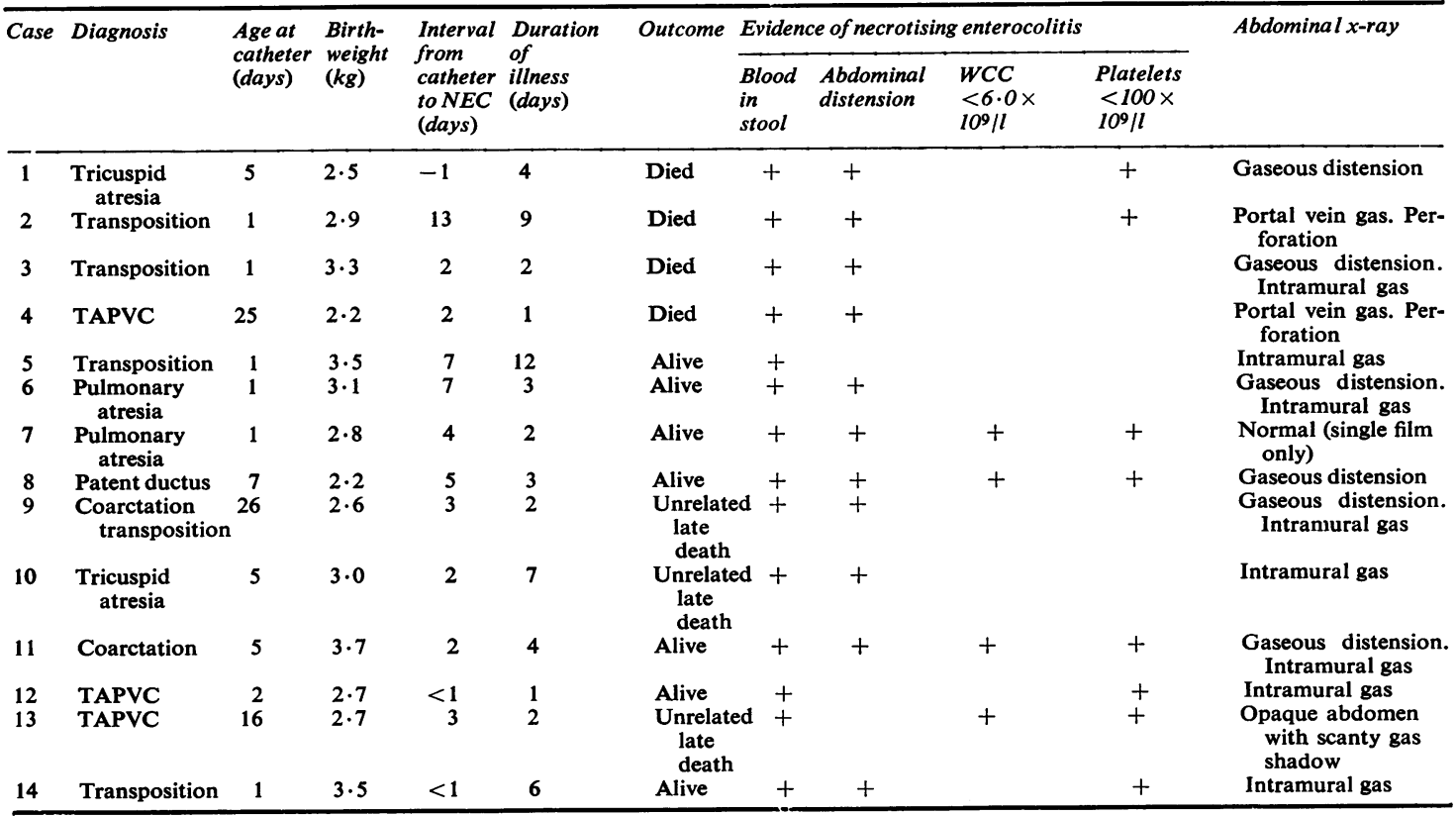

TAPVC $=$ total anomalous pulmonary venous connection.

Table 2 Comparison between 14 patients who developed necrotising enterocolitis, and 97 who did not. No statistically significant differences between the two groups were present

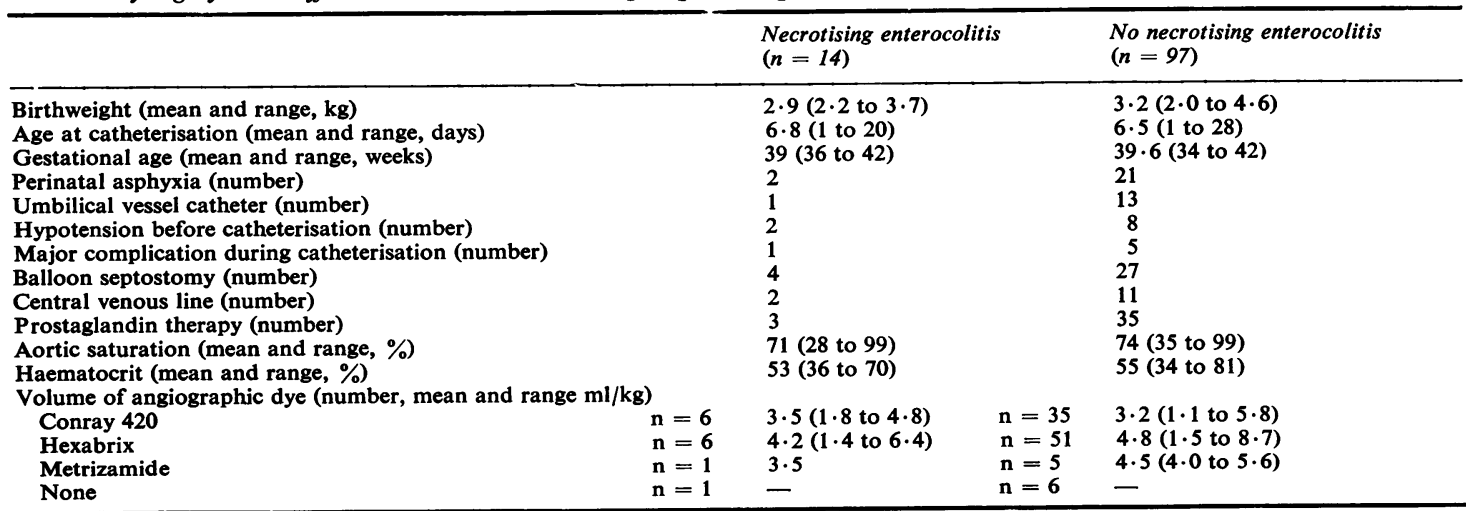

during the period NEC was an important cause of morbidity and mortality among neonates catheterised in this unit. Whether NEC represents a specific complication of cardiac catheterisation or whether it is a chance association in a group of sick neonates is unclear. No case of NEC occurred in infants referred to the unit who were not catheterised but such infants are not comparable with the infants reported here because of the less severe haemodynamic disturbance caused by their cardiovascular problem. In 8 patients a reasonably close temporal relationship between catheterisation and the onset of NEC was apparent, but in one the first indication of NEC preceded investigation by several hours and in 5 NEC developed late, 4 to 13 days after catherisation. The patients who developed NEC had cardiac defects representative of the spectrum of patients investigated in this unit as neonates ${ }^{5}$ and an association between NEC and major cyanotic cardiac defects cannot be inferred. 
The pathogenesis of NEC is imperfectly understood but injury to the bowel mucosa and the subsequent entry of gas-forming organisms into the bowel wall are presumed to be important factors. Hypoxia, hypotension, and local vascular changes are potential causes of mucosal injury and a history of perinatal asphyxia and the use of umbilical vessel catheters have been shown to be significantly associated with the development of NEC in preterm infants. ${ }^{6}$ An analysis of several known and other possible predisposing factors to NEC in our cases showed no significant differences between the two groups and the cause of NEC among these patients therefore remains unknown.

The angiographic contrast medium formerly used during cardiac catheterisation in this unit, sodium iothalamate (Conray 420), has an osmolality of $2100 \mathrm{mmol} / \mathrm{l}$. Cooke et al. ${ }^{4}$ suggested that the hyperosmolar effect of the injection of contrast medium might result in damage to the colonic mucosa leading to NEC and that the use of contrast media with a lower osmolality might avoid this complication. Regrettably this has not been the case and the frequency with which NEC developed in patients investigated using meglumine/sodium ioxaglate (Hexabrix, osmolality $600 \mathrm{mmol} / \mathrm{l})$ and metrizamide (Ampique, osmolality $480 \mathrm{mmol} / \mathrm{l}$ ) was similar to that in patients who received sodium iothalamate. It is possible that toxic substances from plastic components of the apparatus may leak out and be responsible for vascular changes in the portal system as has previously been suggested in cases of NEC after exchange transfusion. ${ }^{7}$ However, the apparatus used was the same for all infants and the duration of cardiac catheterisation is short when compared with an indwelling umbilical vessel catheter. It therefore seems unlikely that this offers a complete explanation for the frequency of NEC in these patients.
In the absence of any recognisable predisposing factors we cannot make any recommendations which might result in a reduction in the prevalence of this problem. Although reports of NEC after cardiac catheterisation are few in number ${ }^{4}$ we feel that it should be recognised as an important association in this age group. In many cases, the illness is fairly mild and similar to that described by Leonidas and $\mathrm{Hall}^{2}$ but this was by no means always the case. However, with appropriate treatment, NEC can be treated successfully, even in the presence of a major cardiac defect and if necessary, emergency cardiac surgery can be performed.

\section{References}

1 Santulli T V, Schullinger J N, Heird W C, et al. Acute necrotizing enterocolitis in infancy; a review of 64 cases. Pediatrics 1975; 55: 376-87.

2 Leonidas J C, Hall R T. Neonatal pneumatosis coli: a mild form of neonatal necrotizing enterocolitis. $J$ Pediatr 1976; 89: 456-9.

3 Polin R A, Pollack P F, Barlow B, et al. Necrotizing enterocolitis in term infants. $J$ Pediatr $1976 ; 89$ : 460-2.

4 Cooke R W I, Meradji M, DeVilleneuve V H. Necrotising entercolitis after cardiac catheterisation in infants. Arch Dis Child 1980; 55: 66-8.

5 Dickinson D F, Arnold R, Wilkinson J L. Outcome of treatment for neonates referred to a supraregional cardiac centre 1976-78. Arch Dis Child 1982; 57: 328-33.

- Bunton G L, Durbin G M, McIntosh N, et al. Necrotising enterocolitis. Controlled study of 3 years' experience in a neonatal intensive care unit. Arch Dis Child 1977; 52: 772-7.

7 Rogers A F, Dunn P M. Letter: Intestinal perforation, exchange transfusion, and PVC. Lancet 1969; ii: 1246.

Correspondence to Dr D F Dickinson, Royal Liverpool Children's Hospital, Myrtle Street, Liverpool L7 7DG.

Received 16 November 1981 